\title{
THE ROLE OF PET CT IN COMPARISON TO TRIPHASIC CT IN EARLY FOLLOW UP OF HEPATOCELLULAR CARCINOMA AFTER TRANSARTERIAL CHEMOEMOBLIZATION
}

\author{
Mounir Sobhy Guirguis*, Enas Ahmed Azzab *, Waleed Mohammed Hetta* and \\ Hany Rafat Atyi El-Malah **
}

\begin{abstract}
* Department of Radiodiagnosis, Faculty of Medicine Ain Shams University.

**El-Sheikh Zayed AL-Nahyan Hospital -Manshet Nasr Ministry of health.
\end{abstract}

Corresponding author:

Hany Rafat Atyia EL -Malah Mobile: 01090069704

E mail:

malah.hany@yahoo.com

Received: 16/4/2019

Accepted: 7/5/2019

\begin{abstract}
:
Background: Hepatocellular carcinoma (HCC) is one of the major causes of mortality among patients with cirrhosis. It represents about $11.85 \%$ of the malignancies of all GIT organs and $1.78 \%$ of the total malignancies among Egyptians.

Aim of the work: This study aimed to compare between the role PET/CT and triphasic CT in evaluation of HCC after trans-arterial chemoembolization (TACE).

Patients and Methods: From November 2016 to November 2018, thirty patients (24 males and 6 females), their age ranged from 44 to 75 years with mean age of 60.6 years were referred to the radiology unit after trans-arterial chemoembolization (TACE) of hepatocellular carcinoma. ${ }^{18} F-F D G$ PET/CT and triphasic CT examinations were conducted in a private radiology center in Cairo for all patients using combined PET-CT machine (Philips intgenuity TF PET/CT 128 slices).The reference standard to determine the accuracy of the results included laboratory serial AFP level monitoring, follow up by different imaging modalities and histopathological results of the resected liver in case of transplantation.
\end{abstract}

Results: PET/CT showed sensitivity, specificity and accuracy of $96.3 \%, 66.7 \%$ and $93.3 \%$ respectively in comparison to $74 \%, 100.0 \%$ and $76 \%$ for triphasic CT.

Conclusion: ${ }^{18}$ F-FDG PET/CT showed higher diagnostic accuracy over triphasic CT in evaluation of intervention bed following TACE regardless the degree of tumor vascularity or lipiodol deposition that could limit detecting residual disease.

Keywords: 18F-FDG PET/CT, HCC, MRI, TACE, AFP

\section{INTRODUCTION:}

Hepatocellular carcinoma (HCC) represents the commonest primary hepatic tumor of adults. It is the $6^{\text {th }}$ most common tumor in the world and the third commonest cause of cancer related deaths ${ }^{(1)}$.

HCC is caused by malignant transformation in hepatocytes due to chronic liver diseases resulting in cirrhosis ${ }^{(2)}$.

From the selective treatment options of liver tumors, interventional procedures such as Trans arterial chemo-embolization (TACE), has been widely used. The powerful cytotoxic effect of TACE by combined action of ischemia, followed by chemoembolization of the tumor's feeding artery has been proved to result in therapeutic efficacy ${ }^{(3)}$.

Despite good results, this interventional procedure needs close monitoring to effectiveness of treatment, because the rate 
of residual viable malignancy in tumors larger than $3 \mathrm{~cm}$ can reach $48 \%{ }^{(2)}$.

Follow up of tumor response after TACE is important to determine whether the tumor is completely eradicated or additional treatment is required. Magnetic resonance imaging (MRI) and computed tomography (CT) have been widely used for the assessment of treatment response after TACE. The determination of treatment response using size criteria, based on the Response Evaluation Criteria in Solid Tumors (RECIST), does not necessarily apply well to interventional therapy in such patients. So most radiologists have relied on the presence or absence of local contrast enhancement at the treated tumor in addition to changes in tumor size ${ }^{(4)}$.

In contrast to morphological image diagnosis, FDG-PET evaluates the viability of HCC based on glucose metabolism, is not influenced by tumor size, morphology or lipiodol deposition ${ }^{(5)}$.

Positron Emission Tomography (PET) uses 18- fluoro-deoxy-glucose (18- FDG) as radioactive material showing difference in metabolism between tissues, thus demonstrates the functional status of suspicious lesions $^{(6)}$.

\section{AIM OF THE WORK:}

The aim of this study is to emphasize the role of PET/CT in early follow up of HCC after Transarterial chemoembolization in comparison to Triphasic CT.

\section{PATIENTS AND METHODS:}

\section{Patients:}

From November 2016 to November 2018 , thirty patients ( 24 males and 6 females), their age ranged from 44 to 75 years with mean age $60.6 \pm 7.35$ years, were referred to the radiology unit with hepatocellular carcinoma and submitted to
TACE. ${ }^{18}$ F-FDG PET/CT followed by triphasic CT examinations were conducted in a private radiology center in Cairo for all patients using combined PET-CT machine (Philips intgenuity TF PET/CT 128 slices).

\section{The patients were subjected to the following:}

-Detailed careful history taking before doing the study especially for allergy or reactions to contrast material.

-Laboratory analysis including serum creatinine and tumor marker (AFP).

\section{Inclusion criteria:}

- Patients of any age who underwent TACE for HCC.

- Both sexes were included.

- Two patients had history of combined TACE and RFA procedures .

- One patient submitted to TACE on top of transplanted liver.

\section{Exclusion criteria:}

- Patients with past history of contrast allergy.

- $\quad$ Patients with blood glucose level $>300$ $\mathrm{mg} / \mathrm{dl}$ at the time of the study.

- High serum creatinine $>2 \mathrm{mg} / \mathrm{dl}$.

- Small lesions $<10 \mathrm{~mm}$.

All patients underwent PET/CT study followed by Triphasic CT in the supine position after a $6 \mathrm{hrs}$ fast. Scans were acquired $60 \mathrm{~min}$ after injection of $1 \mathrm{mCi} / 10$ $\mathrm{kg}$ of 18FDG. A low dose non contrast CT acquired for attention correlation and anatomic localization followed by PET scan covering a field of view starting from the skull base to the mid-thigh level followed by injection of I.V. non -ionic contrast (with dose $2 \mathrm{mg} / \mathrm{kg}$ patient body weight) and then a diagnostic triphasic CT examination of the same regions was done.

Viable HCCs were identified by 
presence of metabolically active tumor tissue with high FDG uptake and correlate this activity to its anatomical site in the corresponding CT images.

The images were interpreted both visually and semi quantitatively for the regions with pathologic FDG accumulation using standardized uptake value (SUV). The SUV is a semi quantitative assessment of the radiotracer uptake from a static (single point in time) PET image .Typically malignant tumors have an SUV more than normal liver background uptake The study was done after approval of ethical board of Ain Shams University and an informed written consent was taken from each participant in the study.

\section{RESULTS:}

To determine the accuracy of the results, our standard included laboratory serial AFP level monitoring; follow up by different imaging modalities and histopathological results of the resected liver in case of transplantation.

Serial AFP monitoring of the 30 included patients were as following; 27 patients had persistent elevated AFP levels and the remaining 3 patients had normal levels of AFP and were preparing for liver transplantation. Out of the 27 patients with elevated AFP levels, triphasic CT study was positive for viable tumoral tissue in only 20 patients and showed no enhancement in 7 patients, among these CT negative patients, PET /CT showed positive nodular FDG uptake at intervention bed in 3 patients and the other 4 patients showed extra hepatic metastatic spread in spite of successful local chemo-embolization. While PET/CT was positive in 26 patients out of the 27 patients with elevated AFP levels, this leaved a single fore mentioned patient by PET/CT which showed characteristic triphasic enhancement pattern of viable HCC. The other 3 patients with normal AFP level (were preparing for liver transplantation) showed no abnormal findings on $\mathrm{CT}$, yet on PET/CT there was a single false positive patient due to regional hyperemia causing increased FDG uptake this patient with regional hyperemia underwent liver transplantation and histopathological of the resected liver revealed complete necrosis with no malignant cells.

Among the patients with positive extra hepatic metastasis detected by PET/CT; one patient showed local recurrence at the intervention bed with metastatic abdominal lymph nodes and 4 patients were positive for extra hepatic metastasis in spite of successful local treatment (two patients were positive for osseous deposits, one for supra renal metastasis and one for portal vein thrombosis).

Table (1): Sites of extra hepatic spread

\begin{tabular}{|l|l|c|c|}
\hline \multicolumn{2}{|c|}{ Sites of extra-hepatic spread } & No. & $\%$ \\
\hline \multirow{2}{*}{ Bone } & Negative & 28 & $93.3 \%$ \\
\cline { 2 - 4 } & Positive & 2 & $6.7 \%$ \\
\hline \multirow{2}{*}{ Suprarenal } & Negative & 29 & $96.7 \%$ \\
\cline { 2 - 4 } & Positive & 1 & $3.3 \%$ \\
\hline \multirow{2}{*}{$\begin{array}{l}\text { Portal vein tumoral } \\
\text { thrombosis }\end{array}$} & Negative & 29 & $96.7 \%$ \\
\cline { 2 - 4 } & Positive & 1 & $3.3 \%$ \\
\hline Lymph nodes & Negative & 29 & $96.7 \%$ \\
& Positive & 1 & $3.3 \%$ \\
\hline
\end{tabular}

The SUVmax of tumor and the ratio of the tumor SUVmax to normal-liver SUVmax (TSUVmax/LSUVmax) were calculated for each patient. The median value of Tumor SUVmax/Liver SUVmax (TSUV max / LSUV max) in the positive cases was 2.9 (ranged from 1.06 to 7.2), most of them were poorly differentiated HCCs, considering that the cutoff TSUV max/ LSUV max value in the current study was 1 .

Triphasic CT showed true positive results in 20 patients, true negative results in 3 patients, false negative results in 7 patients and no false positive patients. PET/CT showed true positive results in 26 patients, true negative results in 2 patients, false negative results at one patient and false positive results at one patient.

In our study, the sensitivity and 
specificity of ${ }^{18}$ F-FDG PET/CT were $96.3 \%$ and $66.7 \%$ respectively. While the sensitivity and specificity of triphasic CT were about 74 $\%$ and $100.0 \%$ respectively.

Chart (1): Illustrating the diagnostic value of FDG PET/CT and triphasic CT in post TACE follow up.

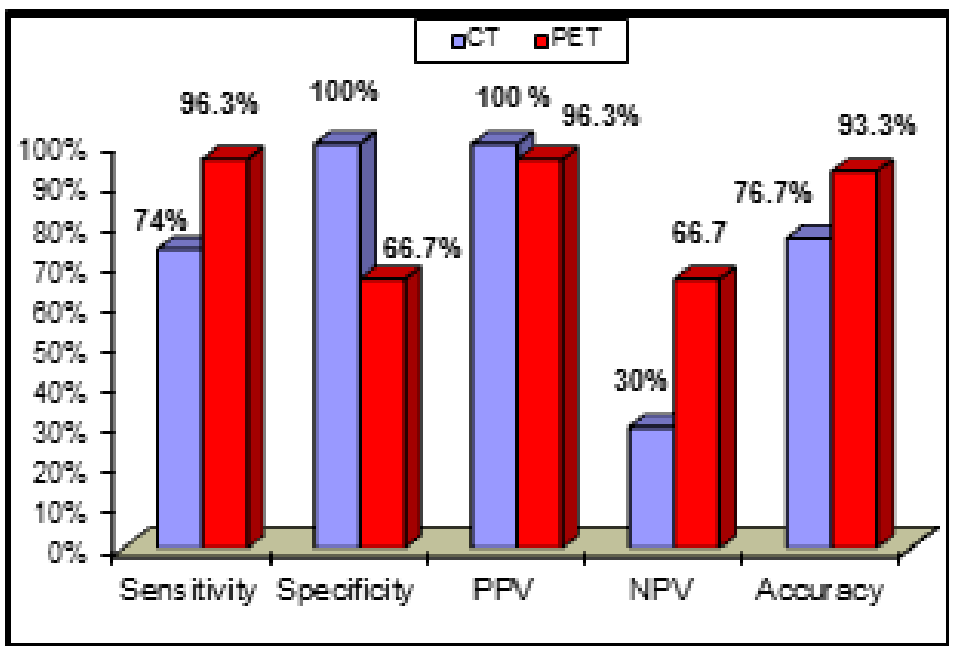

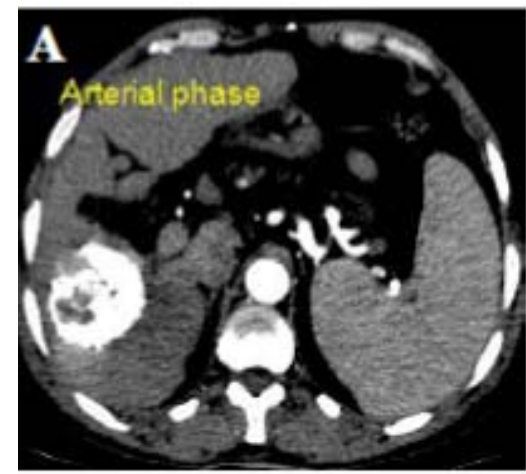

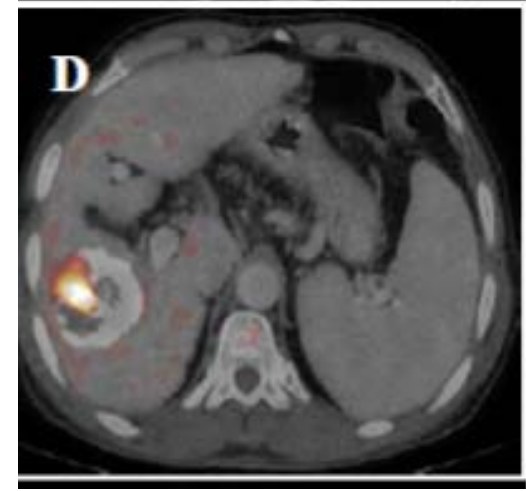

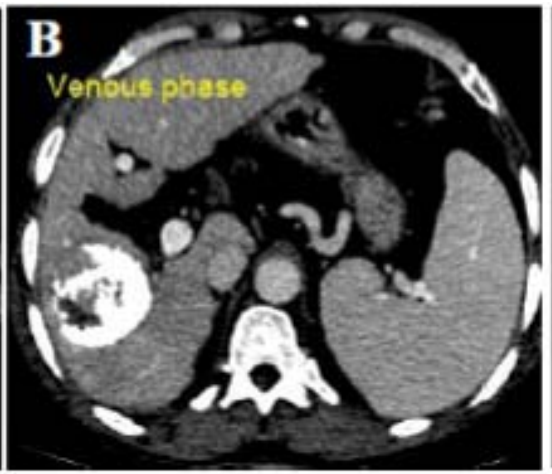

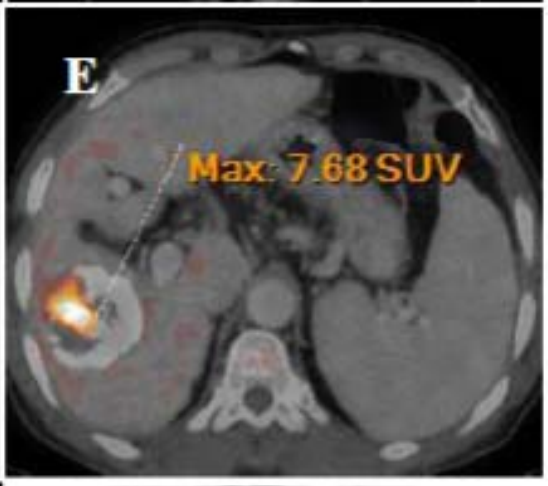

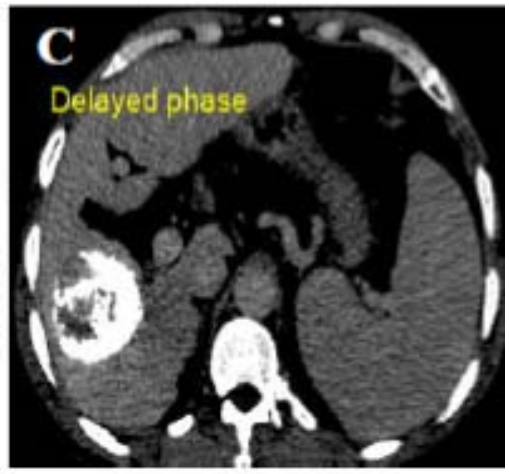

$\mathbf{F}$

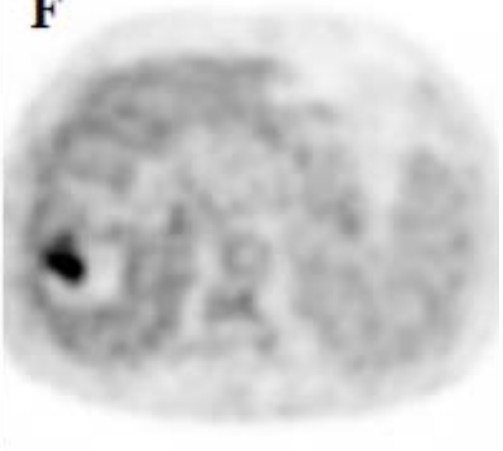

Fig. (1) Fifty seven years old male patient, presented with history of chemoembolization for hepatic focal lesion and persistent elevated alpha fetoprotein level. A, B, C CECT phases show dispersed lipiodol hindering accurate assessment. $\mathbf{D}$ and $\mathbf{E}$ fused PET/CT image showing hyper metabolic activity at right superior margin of the chemo-emoblized lesion. F Axial CTAC image showing focal FDG uptake 

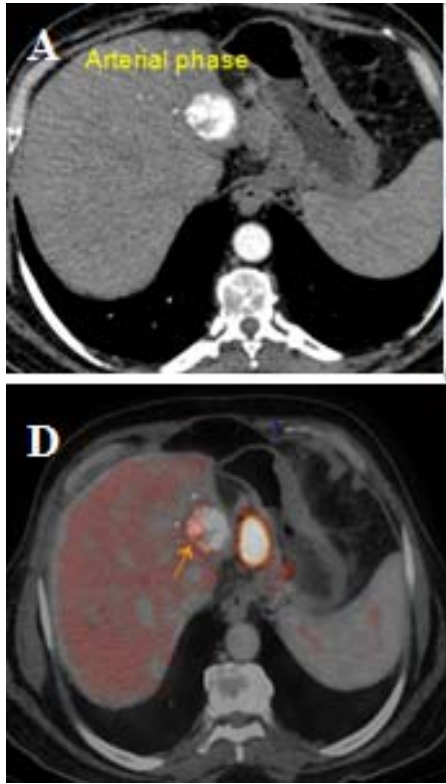
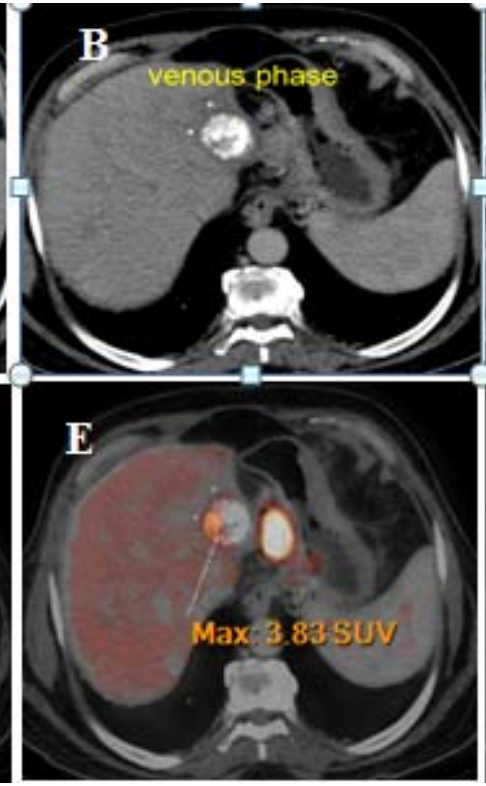

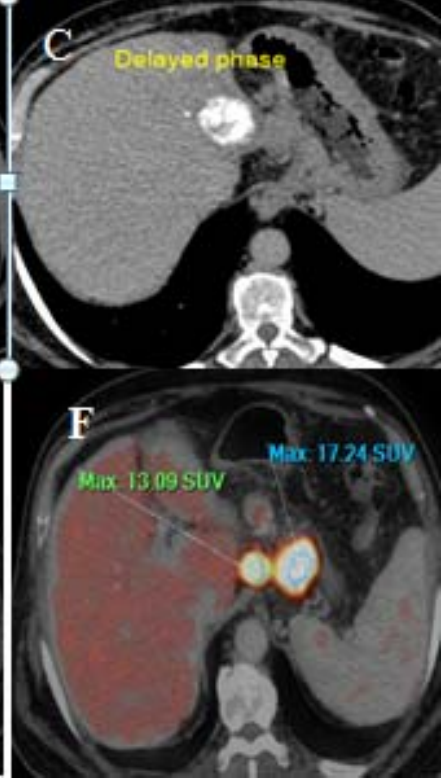

Fig. (2) Sixty years old male patient, presented with history of liver cirrhosis and hepatic focal lesion for which TACE was done and serial AFP level was raising. A, B, C CECT phases show no abnormality with good lipiodol concentration however at $\mathbf{D}$ and $\mathbf{E}$ fused PET/CT image, hyper metabolic focal uptake at lateral aspect of the chemo-emoblized lesion. F Axial fused PET CTT images showhypermetabolic metastatic upper abdominal L.Ns.
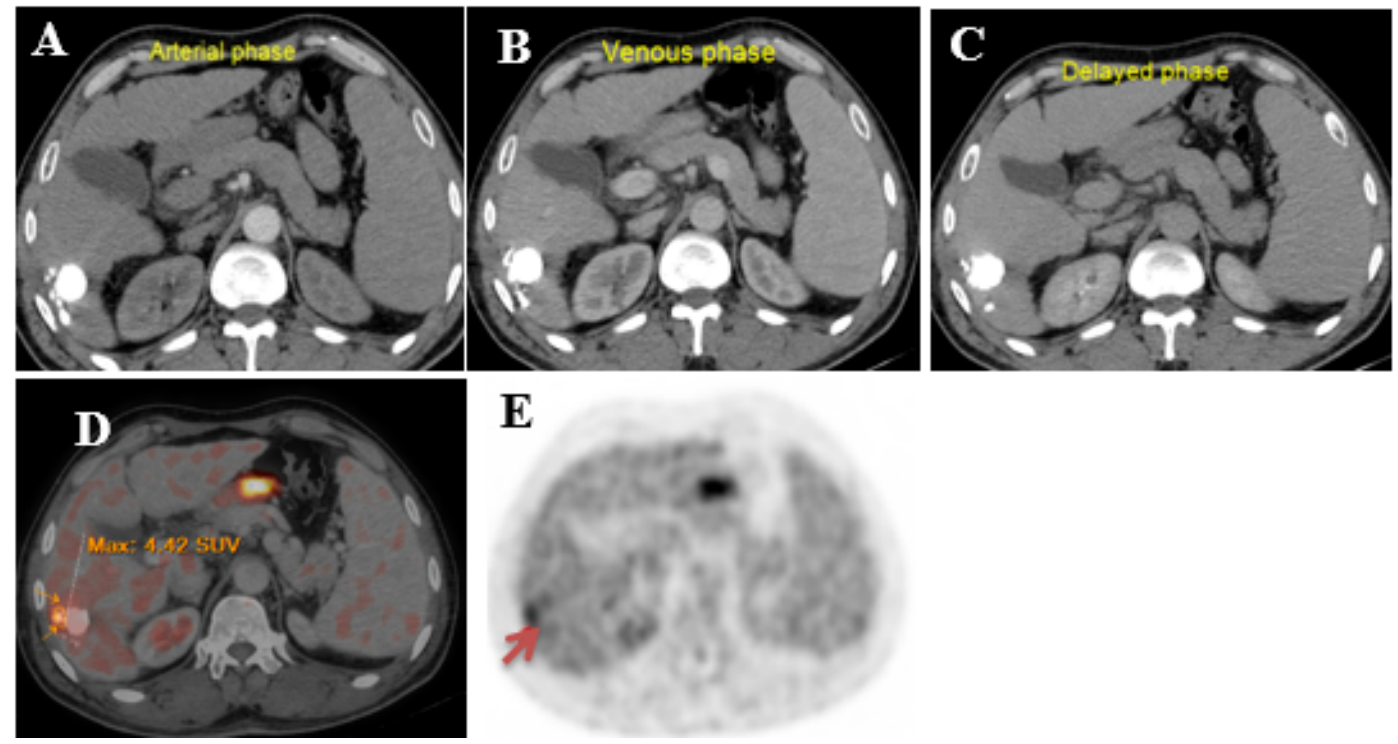

Fig. (3): Fifty seven years old male patient with history of chemo-embolization for hepatic focal lesions, presented with elevated alpha fetoprotein. A,B,C CECT phases show no abnormality with good lipiodol concentration however at $\mathbf{D}$ fused PET/CT image, there is focal increase FDG uptake at the periphery of the embolized lesion at segment VI. E Axial CTAC image showing the focal FDG uptake around the emoblized lesion (red arrow). 

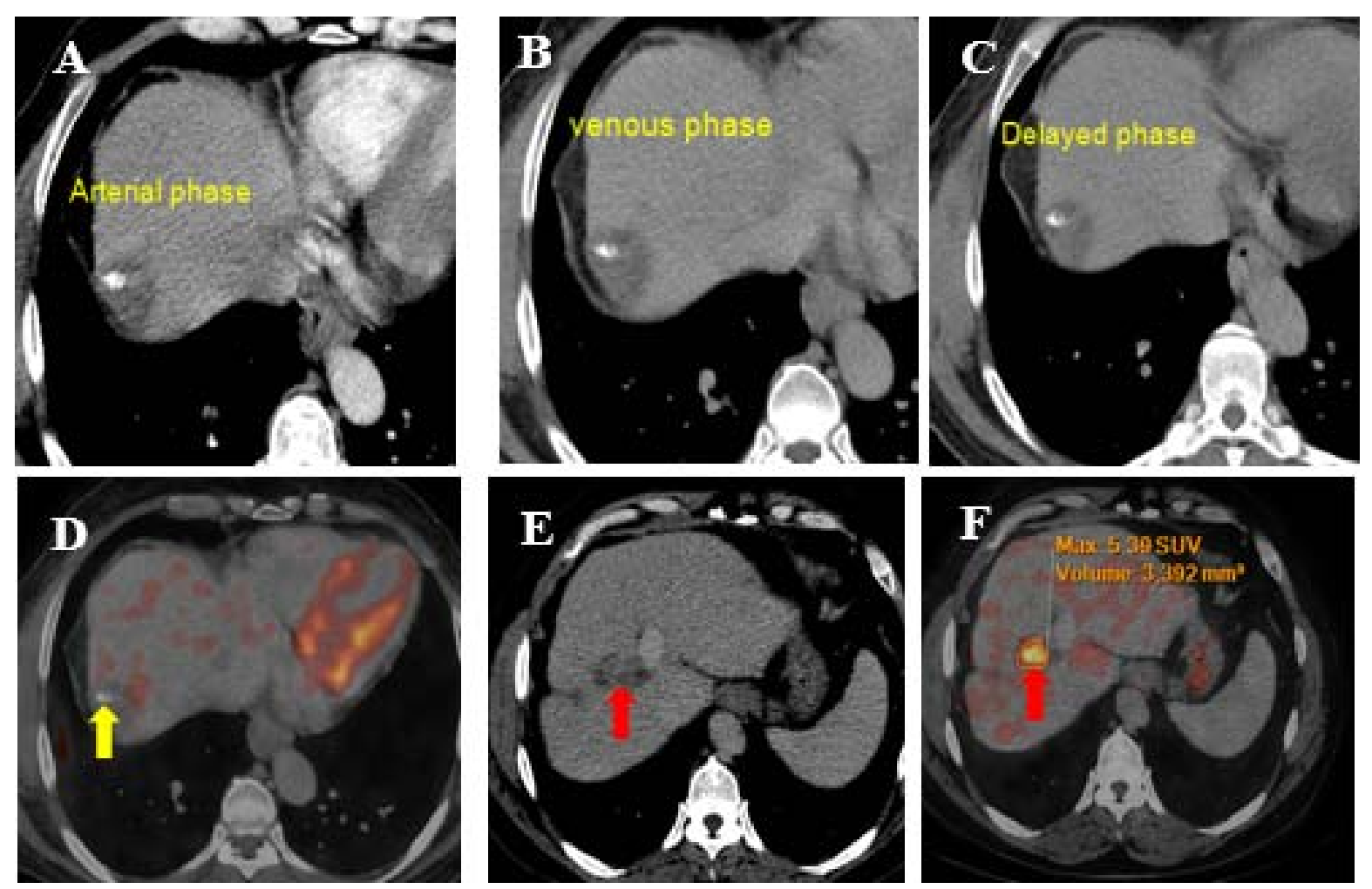

Fig. (4): Sixty eight years old male patient, presented with elevated alpha fetoprotein serum level (213 $\mathrm{ng} / \mathrm{mL}$ ) and had history of HCC treated by TACE. PET/CT was needed for assessment.A, B, C CECT phases show no abnormality at site of TACE. D fused PET/CT images well ablation of the chemoemoblized lesion (yellow arrow). E Axial CECT shows portal vein thrombus (Red arrow). F axial fused PET/CT image shows hyper metabolic portal vein thrombus denoting tumoral thrombus other than benign PV thrombus.

\section{DISCUSSION:}

Hepatocellular carcinoma is considered one of the most common malignant tumors worldwide. Early detection and treatment of recurrent HCC after loco regional interventional treatment are important to patient survival.

Percutaneous techniques such as TACE are now available to manage localized HCC. Assessment of tumor response after TACE is important to determine whether the tumor is completely eradicated or needs additional treatment.

CT and MRI are the most widely tools to assess patients underwent loco-regional intervention procedures such as TACE based on reduction in size or changes in the internal structure as well as enhancement pattern.

The evaluation of treatment response using size Criteria, based on the Response Evaluation Criteria in Solid Tumors (RECIST), does not apply well post chemoembolization of HCC. So most investigators have depended on the presence or absence of contrast enhancement for determination of therapeutic response ${ }^{(7)}$.

The beam-hardening artifact of highattenuation lipiodol on CT can mask the intra-lesional viable tumor detection, in addition after TACE the feeding arteries of the residual tumor were significantly thinner; these changes may affect the degree of enhancement of the tumor.

$\mathrm{PET} / \mathrm{CT}$ is unique combination of the cross-sectional anatomic data provided by 
$\mathrm{CT}$ and the metabolic data provided by PET .In contrast to morphological image diagnosis, FDG-PET evaluates viability based on glucose metabolism, is not influenced by tumor morphology or lipiodol deposition $^{(2)}$.

In agreement with Ali et al., 2016, ${ }^{(8)}$ our study ensured that the chemo-emoblized lesions that become completely photopenic immediately after embolization is suggestive of successful ablation while focal, nodular and intense uptake of FDG within the ablated zone is suggestive of residual viable HCC while a uniform low-grade FDG uptake depicted in the periphery of the ablated lesion is suggestive of reactive tissue changes as inflammation and hyperemia.

The avidity of FDG uptake was defined in our study as maximum standardized uptake value (SUV max). Positive FDG uptake is considered when the liver lesion uptake is higher than the physiological background activity in the surrounding normal liver tissue. (SUV) was measured by the region of interest (ROI) technique.

In our study the median value of TumorSUVmax in the positive cases was 7.4 (ranged from 3.5 to 24), most of them were poorly differentiated $\mathrm{HCCs}$, yet with a single case measuring about 1.4 SUVmax (of well differentiated HCC type). In the study done by Ahn et al. $(2011)^{(9)}$ the median value of Tumor-SUVmax 4.3 (ranged from 2.0 to 11.6).

Kim et al. (2010) ${ }^{(15)}$ stated that, HCCs with high ${ }^{18} \mathrm{~F}$-FDG uptake are reported to be more aggressive than HCCs with low ${ }^{18} \mathrm{~F}$ FDG uptake and this is concordant with $\mathrm{Ho}$ et al. (2007) ${ }^{(12)}$ who stated that poorly and moderately differentiated HCC have lower levels of glucose-6-phosphatase enzyme (which is responsible for rapid clearance of FDG-6-phosphate from hepatocytes) and higher levels of hexokinase, leading to intracellular trapping of FDG causing avid FDG uptake of these tumors on PET and this also was noted in our study where almost all positive cases of HCC were of moderately or poorly differentiated types, showing increased FDG uptake and SUV max value $>3$, while a single case of well differentiated type was encountered in our study showing relatively low ${ }^{18}$ F-FDG uptake and 1.4 SUVmax value.

Small lesions $<10 \mathrm{~mm}$ that are below the scanner resolution might be missed unless they show avid FDG uptake on top of limited background activity so we exclude lesion less than $10 \mathrm{~mm}$ from our study.

The diameter of the lesions in our study ranged between $1 \mathrm{~cm}$ and $8.5 \mathrm{~cm}$ (with mean diameter of $4.2 \mathrm{~cm}$ ) which is comparable to the study done by Ahn et al. (2011) ${ }^{(9)}$ where the diameter of the lesions ranged between 2.5 and $10.5 \mathrm{~cm}$ with mean diameter of 5.5 $\mathrm{cm}$.

The characteristics of portal vein tumoral thrombosis (PVTT) consist of linear avid FDG uptake along the portal vein thrombus, contrast enhancement and expansion of the involved portal vein Hanajiri et al. $(2005)^{(11)}$ found that $18 \mathrm{~F}$ FDG PET was more sensitive than conventional $\mathrm{CT}$ and MRI in detecting suspected vein tumor in patients with HCC also $\mathrm{Hu}$ et al. (2014) ${ }^{(14)}$ investigated the value of FDG PET/CT scan in differentiating between malignant and benign portal vein thrombus with sensitivity, specificity and accuracy of $93.6 \%, 80.0 \%$ and $88.9 \%$ respectively.

Our study demonstrated higher sensitivity of PET/CT over Triphasic CT in detection of local tumor residue /recurrence following TACE as well as detection of extra hepatic spread of HCC in a single whole body examination which may be crucial for patient preparing for liver transplantation. PET/CT showed sensitivity, specificity and accuracy of $96.3 \%, 66.7 \%$ and $93.3 \%$ respectively in comparison to $74 \%, 100.0 \%$ and $76 \%$ for Triphasic CT. These results are 
comparable to many studies as Song et al. $(2015)^{(10)}$ who reported that PET /CT sensitivity, specificity and accuracy for detection of viable HCC after TACE were $89.29 \%, 65.71 \%$ and $80.22 \%$ respectively in comparison to $60.71 \%, 77.1 \%$, and $67.03 \%$ for CECT also Kim et al. (2012) ${ }^{(7)}$ stated that the respective values for sensitivity, specificity and accuracy of PET/ CT in the evaluation of early treatment response after interventional therapy for Hepatocellular Carcinoma were $87.5 \%, 71.4 \%$ and $80.0 \%$.

Our results are also correlated with the results of Jinpeng et al. (2013) ${ }^{(13)}$ in which he studied the recurrence of HCC after TACE in 29 patients, the sensitivity of the PET was $95.4 \%$ while the sensitivity of CECT was $63.8 \%$.

Our results are correlated with Kim et al. $(2010)^{(15)}$ who studied evaluation of metabolic characteristics and viability of lipiodolized hepatocellular carcinomas using18F-FDG PET/CT with sensitivity and specificity $97 \%$ and $63 \%$ for $\mathrm{PET} / \mathrm{CT}$ in comparison to $87 \%$ and $100 \%$ for CECT respectively also Ali et al. $(2016)^{(8)}$ studied the Role of 18-F FDG-PET/CT in the detection of local tumor residue/Recurrence in hepatocellular carcinoma (HCC) post hepatic therapeutic intervention on 40 patients, the sensitivity of triphasic $\mathrm{CT}$ and PET were $76.7 \%$ and $96.7 \%$ respectively .

Conclusion:

So we can conclude that $18 \mathrm{~F}-\mathrm{FDG}$ PET/CT PET/CT showed higher accuracy over triphasic CT in assessment of the intervention bed post chemoembolization of HCC without affection by lipiodol artifact as well as detection of extra hepatic spread of HCC in single whole body examination which is crucial for patients preparing for liver transplantation and differentiation between benign and malignant portal vein thrombosis. However, a larger number of patients and longer term study are still needed to validate the results of this study.

\section{REFERENCES:}

1. Dai L, Ren P, Liu M, Imai H, Tan EM and Zhang JY. Using immunomic approach to enhance tumor-associated autoantibody detection in diagnosis of hepatocellular carcinoma. ClinImmunol. (2014); 152: 127139.

2. Tsurusaki M, Okada M, Kuroda $H$, Mastsuki M, Ishii $K$ and Murakami $T$. Clinical application of 18Ffluorodeoxyglucose positron emission tomography for assessment and evaluation after therapy for malignant hepatic tumor. $\mathbf{J}$ Gastroenterol. (2014); 49: 46-56.

3. Song MJ, Bae SH, Lee SW, Song DS, Kim HY, Yoo IR, Choi JI and Lee YJ. 18FFluorodeoxyglucose PET/CT predicts tumour progression after transarterial chemoembolization in hepatocellular carcinoma. Eur J Nucl Med Mol Imaging. (2013); 40: 865-873

4. Kim KW, Lee JM and Choi BI. Assessment of the treatment response of HCC. Abdom Imaging. (2011); 36: 300-14.

5. Wang $X$, Chen $D$ and Zhang $X$.Value of FDG-PET/CT in the detection of recurrent hepatocellular car-cinoma after hepatectomy or radiofrequency ablation. Chinese society of gastroenterology August (2013); 14 (8): 433- 8.

6. Saif MW, Tzannou I, Makrilia $N$ and Syrigos K. Role and cost effectiveness of $\mathrm{PET} / \mathrm{CT}$ in management of patients with cancer. Yale J Biol Med. (2010); 83: 53-65.

7. Kim SH, Won KS, Choi BW, JO I, Zeon SK, Chung WJ and Kwon JH. Usefulness of F18 FDG PET/CT in the Evaluation of Early Treatment Response after Interventional Therapy for Hepatocellular Carcinoma. Nucl Med Mol Imaging. (2012); 46: 102110.

8. Ali MI, Azab AO, El-Refaei SM, Houseni $M M$ and Hawana MA. Role of 18-F FDGPET/CT in the Detection of Local Tumor Residue/ Recurrence in Hepatocellular Carcinoma (HCC) Post Hepatic Therapeutic 
Intervention. Med. J. Cairo Univ. (2016) September; Vol. 84: No. 1: 991-998.

9. Ahn SG, Kim SH and Jeon TJ.The role of preoperative $\left[{ }^{18} \mathrm{~F}\right]$ fluorodeoxy glucose positron emission tomography in predicting early recurrence after curative resection of hepatocellular carcinomas. J Gastrointest Surg. (2011) Nov; 15(11):2044-52.

10. Song HJ, Cheng JY, HU SL, Zhang GY, FU $Y$ and Zhang $Y J$. Value of $18 \mathrm{~F}-\mathrm{FDG}$ $\mathrm{PET} / \mathrm{CT}$ in detecting viable tumour and predicting prognosis of hepatocellular carcinoma after TACE. Clinical Radiology. (2015); 70. 128e137.

11. Hanajiri $K$, Mitsui $H$ and Maruyama T.18F-FDG PET for hepatocellular carcinoma presenting with portal vein tumor thrombus. J Gastroenterol. (2005); 40: 1005-1006.

12. Ho $C L$, Chen $S$ and Yeung DW.Dual-tracer $\mathrm{PET} / \mathrm{CT}$ imaging in evaluation of metastatic hepatocellular carcinoma. J Nucl Med. (2007); 48: 902-9.

13. Jinpeng L, Wenbo $S$ and Jinlong S. The ther-apeutic effect of transcatheter arterial thromboembolization of hepato-cellular carcinoma as for residual viable tumors related to lipiodol density areas detected by 18F-FDG PET/CT and CT. Hell J. Nucl. Med. (2013); 16 (1): 64-5.

14. Hu S, Zhang J, Cheng C, Liu Q, Sun G and Zuo $C$. The role of F-FDG PET/CT in differentiating malignant from benign portal vein thrombosis. Abdom Imaging. (2014); 39 (6): 1221-7.

15. Kim HO, KIM JS, Shin YM, Ryu JS, Lee YS and Lee SG. Evaluation of metabolic characteristics and viability of lipiolized hepatocellular carcionmas using 18 F-FDG PET/CT. J Nucl Med. (2010); 51: 18491856. 


\section{Mounir Sobhy Guirguis, et al.,}

دورالتصوير الطبقى بالبوزيترون المنبعث المدمج مع الأشعة المقطعية فى المتابعه المبكره لسرطان الخدان الخلايا الكبديه بعد الحقن الكيماوى الثريانى للورم مقارنة بالأشعة المقطعية ثلاثية المراحل

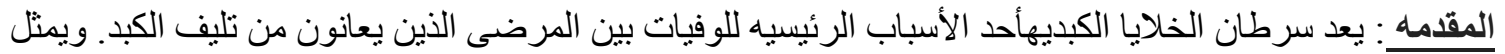

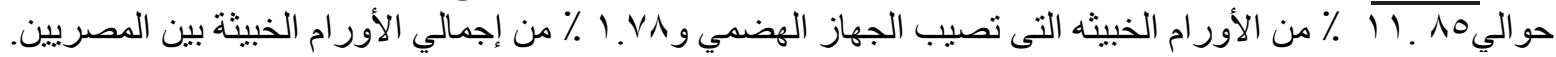

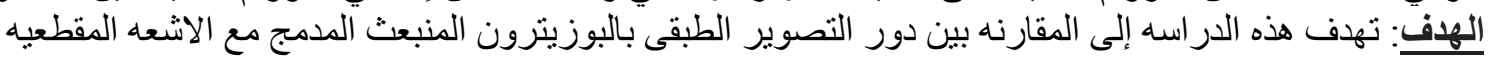

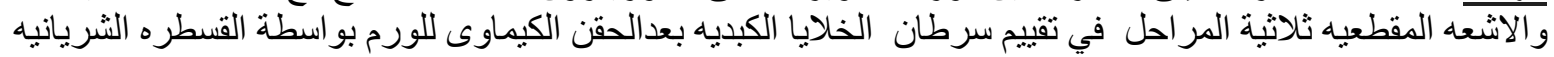
الكبديه.

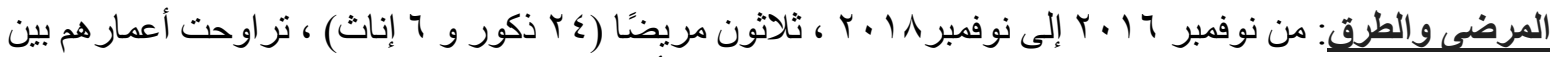

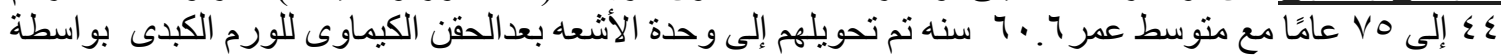

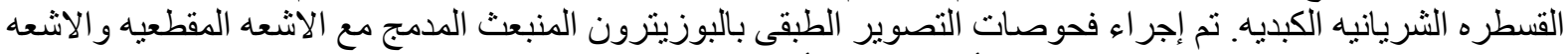

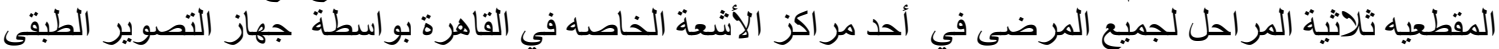

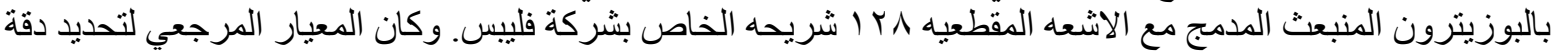

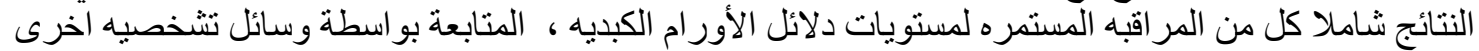

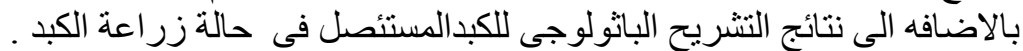

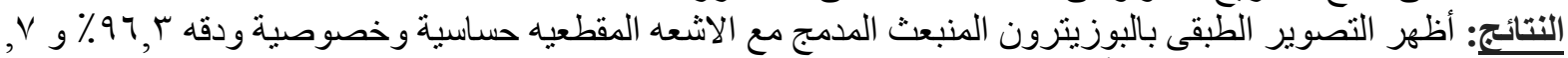

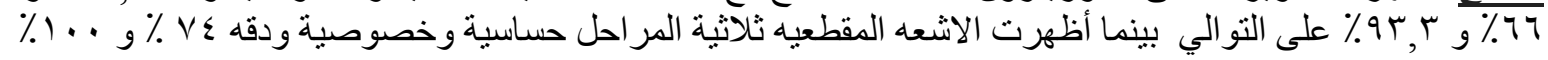

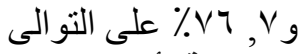
الخلاصة: أظهر التصوير الطبقى بالبوزيترون المنبعث المدمج مع الاشعه المقطعيه دقه أعلى من الاشعه المقطعيه ثلاثية

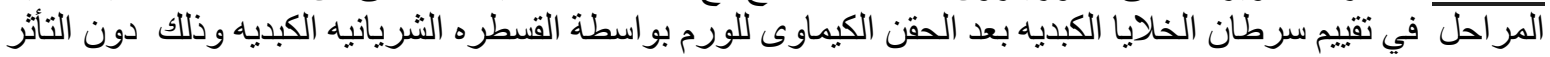
بدرجة الإمداد الدموى للورم او ترسيب الليبدول و هو ما قد يحد من الكثف عن الإنف الاور ام المتبقيه. 